\title{
Charge and Phase Fluctuations in Attractive Hubbard Model
}

\author{
Grzegorz Litak ${ }^{1}$ \\ Department of Mechanics, Technical University of Lublin, Nadbystrzycka 36, \\ PL-20-618 Lublin, Poland
}

\begin{abstract}
Using the negative $U$ Hubbard model we analyze normal state properties of a superconductor. In this model there exists a characteristic pairing temperature $T_{P}$ above a superconducting critical temperature. Below $T_{P}$ electrons start to form incoherent pairs. The fluctuations in charge and phase are precursors of charge density wave and superconductivity phases depending on band filling. They lead naturally to pseudogap opening in the density of states.
\end{abstract}

Key words: fluctuations, pseudogap, CDW

PACS: 74.20.-z, 74.25.-q, 74.40.+k, 71.45.Lr

The pseudogap in electronic spectra of HTc superconducting cuprates has attracted a lot of attention last time [1]. The discussion on its origin and nature is not closed [2] however one of possible explanations could be that it is a precursor of superconducting gap formation $[2,3]$. In this paper we will follow that way analyzing the phase fluctuations of superconducting order [4] parameter mediated by fluctuations of charge.

In this paper we employ the simplest purely electronic model which can lead to superconductivity, namely, the negative $U$ Hubbard model [5]:

$$
\hat{H}=\sum_{i j \sigma}\left(-\mu \delta_{i j}-t_{i j}\right) \hat{c}_{i \sigma}^{+} \hat{c}_{j \sigma}+\frac{1}{2} U \sum_{i} \hat{n}_{i \sigma} \hat{n}_{j-\sigma},
$$

where $i$ and $j$ label the sites of a square lattice, $t_{i j}$ are electron hopping integrals between nearest neighbour sites, $U<0$ describe attraction between electrons occupying the same site $i, \mu$ is the chemical potential. The self-

$\overline{1 \text { Fax: }}+48-815250808$; E-mail: litak@archimedes.pol.lublin.pl 

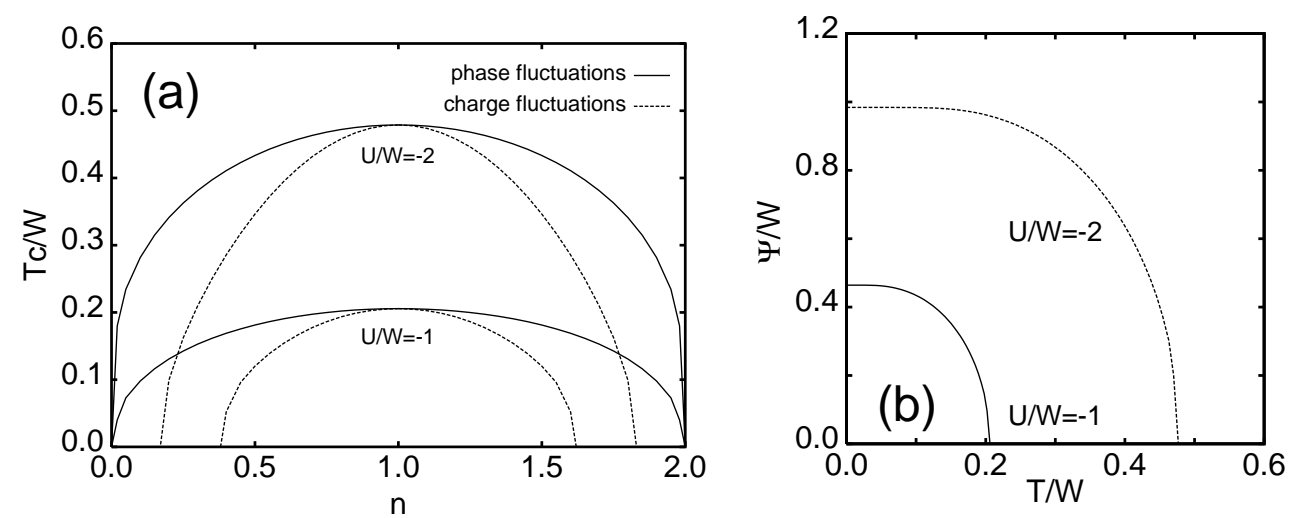

Fig. 1. (a) Characteristic temperature $T_{C}$ for short range ordering: local phase and charge local fluctuations versus band filling $n$, where $W=8 t$ is a band width. (b) Short range orderer parameter $\Psi$ for half filled band $(n=1)$.

consistent Hartree-Fock-Gorkov (HFG) equation:

$$
\sum_{j}\left(\begin{array}{cc}
\left(E+\mu-\frac{U n_{i}}{2}\right) \delta_{i j}+t_{i j} & \Delta_{i} \delta_{i j} \\
\Delta_{i}^{*} \delta_{i j} & \left(E+\mu+\frac{U n_{i}}{2}\right) \delta_{i j}-t_{i j}
\end{array}\right) \mathbf{G}(j, k ; E)=\mathbf{1} \delta_{i k} \cdot(2)
$$

In this case the superconducting order parameter $\Delta_{i}$ and the local charge $n_{i}$ at a finite temperature $T(\beta=1 / k T)$ are given by following relations:

$$
\Delta_{i}=-\frac{U}{\pi} \int_{-\infty}^{\infty} \mathrm{d} E \frac{\operatorname{Im} G^{12}(i, i ; E)}{\mathrm{e}^{\beta E}+1}, \quad n_{i}=-\frac{2}{\pi} \int_{-\infty}^{\infty} \mathrm{d} E \frac{\operatorname{Im} G^{11}(i, i ; E)}{\mathrm{e}^{\beta E}+1} .
$$

To go beyond the HFG we apply fluctuations of phase [4] and charge via Hubbard III approximation [6]. Thus random local pairing potential gains different phase $\Theta_{i}, \Delta_{i} \rightarrow \Delta_{i}\left(\Theta_{i}\right)=|\Delta| \mathrm{e}^{i \Theta_{i}}$ dependent on lattice site $i$. The charge also changes randomly $n_{i} \rightarrow n_{i}=n \pm \delta n_{i}$ with lattice site $i$. In such approach the equation of motion has the following form:

$$
\sum_{j}\left(\begin{array}{cc}
\left(E+\mu-\frac{U n_{i}}{2}\right) \delta_{i j}+t_{i j} & |\Delta| \mathrm{e}^{\mathrm{i} \Theta_{i}} \delta_{i j} \\
|\Delta| \mathrm{e}^{-\mathrm{i} \Theta_{i}} \delta_{i j} & \left(E+\mu+\frac{U n_{i}}{2}\right) \delta_{i j}-t_{i j}
\end{array}\right) \mathbf{G}(j, k ; E)=\delta_{i k} .
$$

Here the local pairing parameter $\Delta_{i}$ and the local charge $n_{i}$ are given by self-consistent relations:

$$
\Delta\left(\Theta_{i}\right)=\frac{-U}{\pi} \int_{-\infty}^{\infty} \mathrm{d} E \frac{\operatorname{Im} G_{\Theta_{i}}^{12}(i, i ; E)}{\mathrm{e}^{\beta E}+1}
$$




$$
\delta n=\frac{n_{A}-n_{B}}{2}=-\frac{1}{\pi} \int_{-\infty}^{\infty} \mathrm{d} \omega \frac{\operatorname{Im}\left(G_{A}^{11}(i, i ; E)\right)-\left(G_{B}^{11}(i, i ; E)\right)}{\mathrm{e}^{\beta E}+1},
$$

where $\bar{\Delta}_{i}=0$ and $\bar{n}_{i}=n\left(n_{A}=n+\delta n\right.$ and $\left.n_{B}=n-\delta n\right)$.

The set of above equations (Eqs. 4-6) can be solved by means of Coherent Potential Approximation (CPA) [4,7]. Single site condition for the coherent potential $\boldsymbol{\Sigma}(i, i, E)=\boldsymbol{\Sigma}(E)$ is defined by the zero value of an average $\mathrm{T}$ matrix:

$$
<\mathbf{T}_{\alpha, \Theta}(i, i ; E)>=<\left(\mathbf{V}_{\alpha, \Theta}-\mathbf{\Sigma}(E)\right)\left(\mathbf{1}-\left[\mathbf{V}_{\alpha, \Theta}-\mathbf{\Sigma}(E)\right] \overline{\mathbf{G}}(i, i ; E)\right)^{-1}>,
$$

where

$$
V_{\alpha, \Theta}=\left[\begin{array}{cc}
\frac{U n_{\alpha}}{2} & -|\Delta| \mathrm{e}^{\mathrm{i} \Theta} \\
-|\Delta| \mathrm{e}^{-\mathrm{i} \Theta} & -\frac{U n_{\alpha}}{2}
\end{array}\right] \quad \text { and } \quad \alpha=A, B, \quad \Theta \in[0,2 \pi]
$$

Equations (5) and (6) can now be expressed through conditionally averaged Green functions:

$$
\left.\mathbf{G}_{\alpha, \Theta}(i, i ; E)\right)=\overline{\mathbf{G}}(i, i ; E)\left(\mathbf{1}-\left[\mathbf{V}_{\alpha, \Theta}-\mathbf{\Sigma}(E)\right] \overline{\mathbf{G}}(i, i ; E)\right)^{-1} .
$$
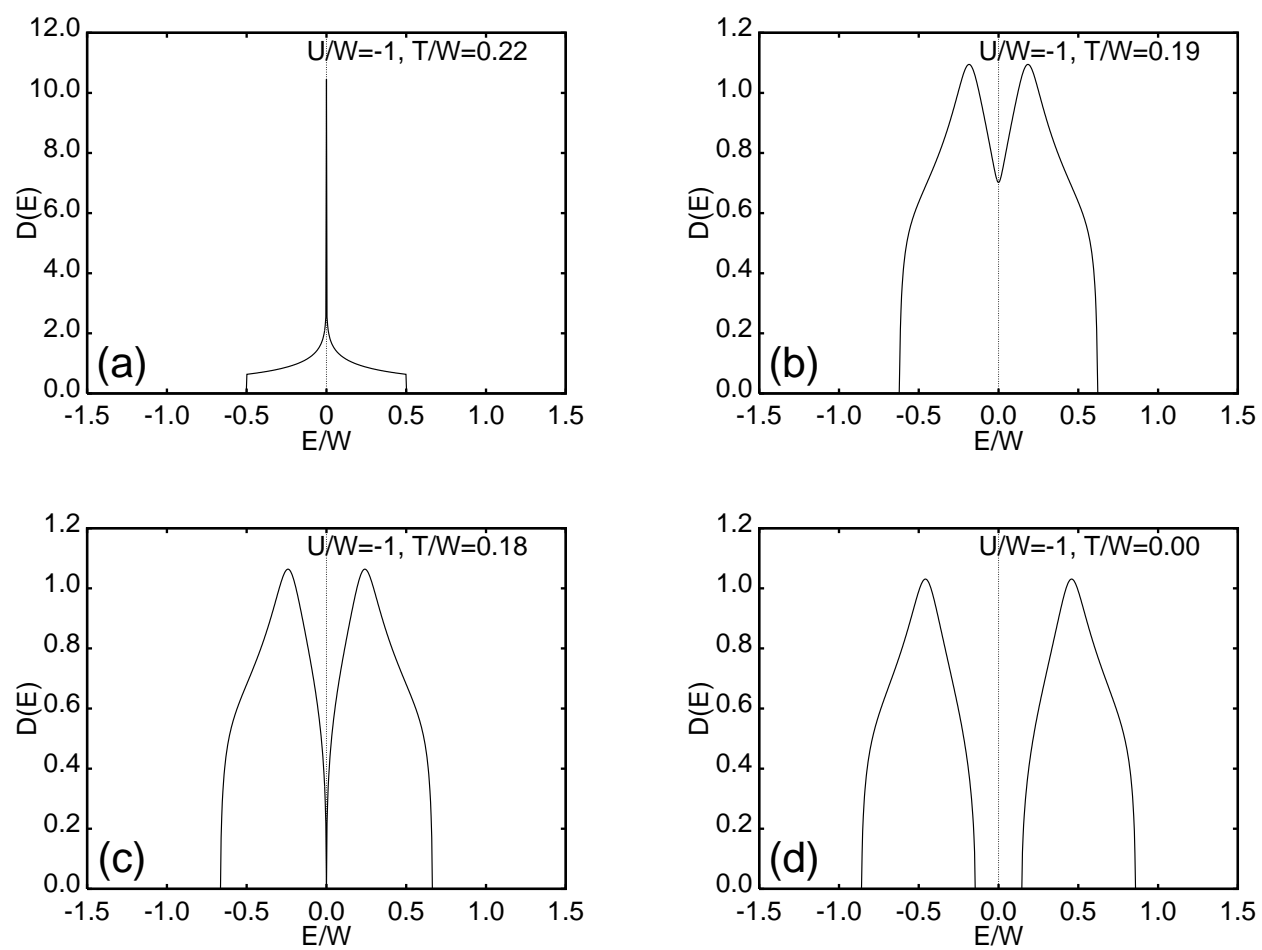

Fig. 2. Average densities at different temperatures and $n=1$. Note, the formation of a pseudogap and a proper gap is caused by fluctuations in charge and phase below the critical temperatures of superconductivity and charge density wave (CDW). 
The above model (Eqs 4-9) has been solved for a square lattice and large enough interaction $|U| / W=1,2$ ( $W=8 t$ is a band width). Fig. 1a shows characteristic temperatures $T_{C}$ of phase (pairing temperature - $T_{P}$ ) and charge fluctuations appearance. Such fluctuations can be naturally related to precursors of long range order phases formation like superconductivity and charge density wave $(\mathrm{CDW})$, respectively. Note that, fluctuations are characterized by different regions of band filling $n$. Charge fluctuations appear around half filled band $(n=1)$ while phase fluctuations are present for any $n$. Interestingly, for particle-hole symmetric situation $(n=1)$ the characteristic temperatures $T_{C}$ are the same for both fluctuations and they can coexist (Fig. 1a). In this case the state can be described by a generalized local order parameter

$\Psi_{i}=\sqrt{|\Delta|^{2}+(U \delta n)^{2}}$ (Fig. 1b). It is similar to low temperature behaviour where the coexistence of superconductivity and CDW [8]. However, in that case the CDW state was not stable in presence of diagonal non-magnetic disorder $[9,10,11]$. The opening of a pseudogap above the superconducting critical temperature $T_{C}($ sup $)$ [1] can be easily explained by such fluctuations. The corresponding densities of states for $U / W=-1$ are presented in Fig. 2. Note that, for smaller temperature $T<0.18 W$ (Fig. 2c) the pseudogap eluate into a proper gap in electronic spectrum. However to investigate further transition into superconducting state one has to investigate Kosterlitz-Thouless scenario [3].

\section{Acknowledgements}

This work has been partially supported by KBN grant No. 5P03B00221.

\section{References}

[1] T. Timusk and B. Statt, Rep. Prog. Phys. 62 (1999) 61.

[2] T. Domański, J. Ranninger, Phys. Rev. B 63134505.

[3] B. Tobijaszewska, R. Micnas, Acta Phys. Pol. A 97 (2000) 393.

[4] B.L. Gyorffy et al., Phys. Rev. B 44 (1991) 5190.

[5] R. Micnas et al., Rev. Mod. Phys. 62, (1991) 113.

[6] J. Hubbard, Proc. R. Soc. London A 277 (1964) 401.

[7] G. Litak et al., Physica C 199 (1992) 191.

[8] P. Miller et al., Physica C 210 (1993) 343.

[9] C. Huscroft and R.T. Scalettar, Phys. Rev. B 55 (1997) 1185.

[10] G. Litak et al., Physica C 308 (1998) 132.

[11] G. Pawłowski and S. Robaszkiewicz, Physica A 299 (2001) 475. 